ity in the microwave background. The discovery of fluctuations, at the expected level of 1 part in 100,000 , meant that the Big Bang was finally a respectable and consistent theory: the last remaining enigma was solved.

The vagaries of space astronomy are also the subject of Ripples in the Cosmos by Michael Rowan-Robinson. The author is professor of astrophysics at Imperial College, London, and has written several popular books on astronomy. He played an important role in the Infrared Astronomical Satellite (IRAS), an infrared space telescope built by a team from the United States, United Kingdom and the Netherlands and launched in 1983. IRAS provided the first all-sky map at infrared wavelengths, and revolutionized our perspective on the cosmos. The most luminous galaxy in the Universe was first seen as an IRAS source. Only in the infrared can one penetrate the cores of interstellar dark clouds, where stars are conceived and born. Only in the infrared can one get an accurate all-sky tally of the large-scale galaxy distribution. This map was used to provide a measure of where in the Universe the gravitational acceleration originates that accounts for the motion of our Galaxy as reflected in the dipole anisotropy in the microwave background. Rowan-Robinson includes a chapter that describes the impact on his life of the ripple announcement.

For the real dirt on COBE, however, I recommend Marcus Chown's slender tome, Afterglow of Creation. The science editor of New Scientist brings a journalistic fervour to delve into the personalities behind the headlines. Here you will read random quotations by the key players about the many rivalries between scientists striving for their share of glory. Both Chown and Rowan-Robinson take care to give proper credit to Arno Penzias and Robert Wilson for accidentally discovering, while employed by Bell Laboratories as young radioastronomers, the microwave background radiation. Chown describes the discovery of the microwave background and the COBE announcement of the ripples, peppered with speculations about Nobel prizes, and why scientists, when stumbling for appropriate words, inevitably need to invoke God for an appropriate metaphor. The public was bombarded with deistic analogies in the post-COBE euphoria, including seeing "the face of God", the "handwriting of God", and discovering the "Holy Grail", not to mention the more secular tribute of "the discovery of the century, if not of all time". Most of the scientists who quoted these words to the media soon came to regret their moment of passion, as a backlash inevitably developed. However, now that the DMR ripples have been confirmed, there is little doubt that the discovery of the cos- mic microwave background fluctuations, as well as the unprecedented precise measurement of its black body spectrum, are a major breakthrough in our understanding of the cosmos. We can reasonably anticipate that appropriate recognition will be made when the attention of the Nobel committee refocuses on astrophysics in a few years' time.

Joseph Silk is in the Astronomy and Physics Departments and Center for Particle Astrophysics, University of California, Berkeley, California 94720, USA.

\section{Physically impossible}

\section{David Goodstein}

Fear of Physics: A Guide for the Perplexed. By Lawrence M. Krauss. Basic Books: 1993. Pp. 206. \$20. (To be published in the UK by Jonathan Cape in 1994 at £17.99).

WHAT we have here is nothing less than an attempt to do the impossible. Fear of Physics, with an apology in the preface to Erica Jong, is subtitled $A$ Guide for the Perplexed, Moses Maimonides unattributed. I'm sure Erica could have taught Maimonides a thing or two, but that's not what this book is about. This is definitely Maimonides, gently introducing Erica to the wisdom of the ages, meaning, of course, contemporary theoretical physics.

Lawrence Krauss, of Case Western Reserve University, has undertaken to explain it all in simple language, almost without committing a single equation. In less than 200 pages, he covers just about everything. Scales, dimensions, little numbers and big numbers and the usefulness of scientific notation; from Galileo to Gell-Mann, from dark matter to critical point fluctuations to the Higgs boson condensate; it's all here (I didn't notice anything on the fractional quantum Hall effect, but I may have dozed off for a few pages). Every bit is explained in clean, simple prose. There is barely a paragraph anywhere that could have been made easier to understand. And yet, sad to say, the enterprise as a whole doesn't quite succeed.

Part of the reason has to do with Krauss. His accounts of physics are technically excellent, but he tends to be sloppy about anything less important to him than physics itself. For example, in one place we are told that Johannes Kepler was Tycho Brahe's student. At a different level of intellectual sloppiness, we are told at another point that Albert Einstein was the Columbus of the twentieth century (you know, the world is round, and spacetime is curved). Another problem is that Krauss feels obliged to intersperse amusing anecdotes in his text, but he has no flair at all for telling them. He chooses the oldest chestnuts around, then delivers them poorly. That includes the one about the theoretical physicist's contribution to dairy farming, the spherical cow. He immediately admits that it's not funny, although he makes it the central metaphor of the book (there's a picture of a cow on the dust jacket - let's hope Erica doesn't misunderstand). I've heard the joke many times and, depending on the skill of the storyteller, sometimes it is funny.

These are quibbles. The real problem is the impossibility of the task itself. Krauss explains point A to us; he can't explain it to us the way he would to an undergraduate class, much less to his own colleagues, because that is not the nature of the undertaking. Instead he surrounds it with metaphors, analogies, simplified examples and so on. When he's done, because he does it very well, we have a pretty good idea of why physicists think point $\mathrm{A}$ is so important, and we may even think we've grasped the point itself.

The problem comes when he starts to explain point $B$, which requires that we already understand point $\mathrm{A}$. He uses all the same strategies just as nicely as before, but now we realize that point $A$ was more slippery than we thought, and point B is really shaky. Then it's on to point $\mathrm{C}$. By the time we get through the entire alphabet pretty much all is lost, except to the unperplexed who had no fear of physics in the first place.

By ironic coincidence, I read Krauss's explanation of the importance of the Superconducting Super Collider on the very day that it died ("Congress finally drove a wooden stake through its heart", said the New York Times). Some day, when history sorts out all these things, it may be decided that the era that ended that day failed for the same reason Krauss's book did. We physicists left the public 300 years behind. The gap has turned out to be too big to fill in.

Richard Feynman, who is one of Krauss's heroes, once had a similar failure. He tried to teach physics to a class of bright first-year college students. $\mathrm{He}$ ended up instead leaving us a set of books that are useless to students but are nevertheless enduring classics of the scientific literature. Fear of Physics is not in that category, but (aside from my quibbles) it is beautifully written and painless to read. Somewhere on the spectrum of scientific sophistication, there must be an audience for this book. I hope the book and the audience find one another.

David Goodstein is in the Department of Physics, California Institute of Technology, Pasadena, California 91125, USA. 